\title{
Preventive reactive power management for improving voltage stability margin
}

\author{
O. Alizadeh Mousavi*, M. Bozorg, R. Cherkaoui \\ École Polytechnique Fédérale de Lausanne, EPFL STI-DEC/GR-SCI, ELL 139, Station 11, 1015 Lausanne, Switzerland
}

\section{A R T I C L E I N F O}

\section{Article history:}

Received 28 November 2011

Received in revised form 16 August 2012

Accepted 10 October 2012

Available online 5 December 2012

\section{Keywords:}

QV-curve

Load reactive power reserve $(L R P R)$

Generator reactive power reserve (GRPR)

Voltage stability margin (VSM)

Complementarity constraint

\begin{abstract}
A B S T R A C T
Voltage stability imposes important limitations on the power systems operation. Adequate voltage stability margin needs to be obtained through the appropriate scheduling of the reactive power resources. The main countermeasures against voltage instability could be distinctly classified into preventive and corrective control actions. This paper proposes a preventive countermeasure to improve the voltage stability margin through the management of the reactive power and its reserve. The voltage and reactive power management is studied from the generator's point of view to maximize effective generator reactive power reserve (EGRPR). Detailed model of the generators including the armature and field current limits, as well as the switch mode between the voltage control and the reactive power limitations are considered to maximize the reactive power capability of the generators in emergency states. One-stage and two-stage optimization approaches are utilized to find the optimum solution. The proposed optimization procedure is applied on a 6-bus system and the New England 39-bus system to illustrate the effectiveness of the method.
\end{abstract}

(c) 2012 Elsevier B.V. All rights reserved.

\section{Introduction}

The voltage and reactive power management has been a concern for power system operators, especially after the restructuring of the power industry. In the restructured environment, the operation of the system is constrained by strict economic constraints. As a result, the network is frequently operated under stress and closer to its operating limits. The evidence of these circumstances is widespread blackouts in the recent two decades. Insufficient voltage and reactive power support was an origin or a factor in the major power outages worldwide [1].

In the context of the electricity market, the voltage and reactive power control service is classified as one of the ancillary services. Until now the system operator is the sole responsible for the management of this critical ancillary service to ensure secure and reliable operation of the system.

Sufficient voltage stability margin (VSM) should be provided to preserve the security of the bulk power system against the short- and long-term instabilities and subsequent voltage degradation and collapse. For this purpose, appropriate control actions should be continuously acquired, deployed and maintained from the control resources. These control actions comprise reactive power reserve (RPR) and emergency countermeasures that can be considered, respectively, as preventive and corrective control actions. The corrective actions include load tap changer blocking,

\footnotetext{
* Corresponding author. Tel.: +4121693 46 61; fax: +41216934662.

E-mail address: omid.alizadeh@epfl.ch (O. Alizadeh Mousavi).
}

capacitor switching, voltage and reactive power rescheduling, then active power rescheduling, and as the last resort load shedding [2]. The main preventive actions against voltage instability are (1) management of reactive power resources through load tap changing, capacitor switching, and (2) implementation of hierarchical or centralized voltage and reactive power control schemes, which both of them affect the RPR. Also, the active power rescheduling can be included in the preventive actions which is not taken into consideration in this paper [3]. Here, the focus is only on the management of the reactive power resources as the most important preventive action.

In order to provide RPR appropriately, both reactive power generation and its reserve should be considered simultaneously in the procurement and the scheduling of the reactive power resources. The RPR can be taken into account from the load or the generator point of view which is called $L R P R$ and GRPR, respectively. The literature paid more attention to $L R P R$ than GRPR and so more investigation is needed for the latter. Moreover, the system operator usually has to manage its reactive power resources for a specified active power dispatch obtained from the active power market. For this purpose, it is assumed that the management of the active and reactive power is decoupled. Furthermore, the increasing interest for the setup of a reactive power market, raise the interest for RPR analysis from the generators' side, since they are the main providers of this service. As a result, this paper focuses particularly on the GRPR.

In this paper, an optimization procedure is proposed for reactive power management considering an operating point correlated to a voltage collapse point to improve the VSM. The aim of the proposed 


(2)

a) Single line diagram of the two bus test system

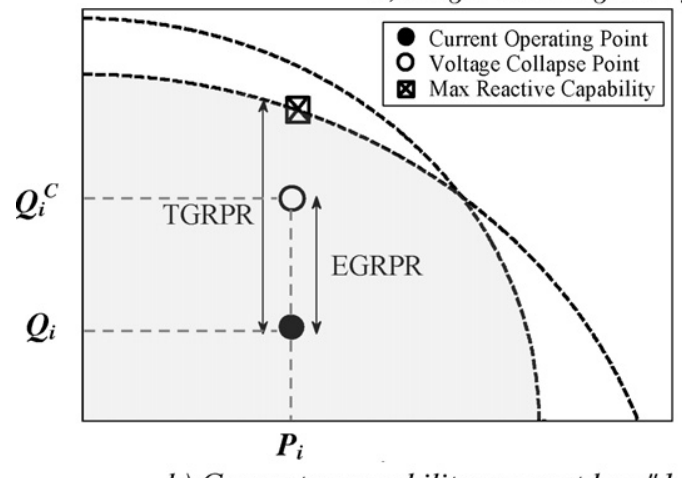

b) Generator capability curve at bus \#1

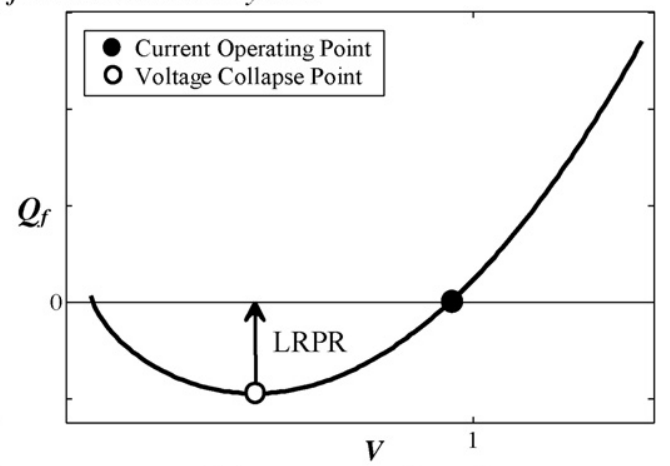

c) QV-curve for bus \#2

Fig. 1. $L R P R, T G R P R$, and EGRPR for the two bus test system.

scheme is to distinguish and to improve the effective RPR of the generators. To deal with it, in Section 2, fundamentals of GRPR and $L R P R$, are discussed more in depth. The proposed reactive power management method regarding VSM is presented based on onestage and two-stage optimization approaches in Section 3. Finally, the proposed method is applied and tested on a 6-bus test system and on the 39-bus New England system. The simulation results and analysis are given in Section 4.

\section{Fundamentals on reactive power reserve}

The RPR is a spare reactive power capability available in the system to assist the voltage control. This capability should be considered to respond to unforeseen events that lead to a sudden change of reactive power requirement. The system operator needs to assign sufficient RPR on the best response resources. Thus, the generators are commonly the main resource of RPR which they are also referred as spinning RPR.

The RPR can be viewed from the load's and the generator's perspective. The two bus test system, shown in Fig. 1a, is used to illustrate the various viewpoints of the RPR. A generator and a load are connected to bus 1 and bus 2, respectively. The $Q V$-curve method, for which more details are given in [4], is used to obtain the reactive power margin to a voltage collapse point. For this purpose fictitious reactive power supports $Q_{f}$ 's are connected to certain load buses referred as pilot nodes. Here, the term "pilot node" is explicitly used for this purpose. The $Q V$-curve, shown in Fig. 1c, expresses the relationship between the reactive power support $\left(Q_{f}\right)$ at the given bus and the voltage $(V)$ at that bus [2]. The minimum point of the $Q V$-curve shows the reactive power margin until the voltage instability. This point is called voltage collapse point and it is indicated by the white circle. The current operating point without compensation $\left(Q_{f}=0\right)$ is indicated by the black circle. The generator reactive power output of the current operating point and the voltage collapse point are shown on the generator capability curve in Fig. 1b. In this paper, the optimal power flow is used to calculate the reactive power margin to the voltage collapse point [5].

The load RPR (LRPR), shown in Fig. 1c, is defined as the minimum amount of the reactive load increase for which the system loses its operability. According to the literature, it is also referred as reactive power margin. The generator RPR (GRPR) focuses on the effectiveness of the provided RPR by each generator. Technical generator RPR (TGRPR), is defined as the difference between the maximum reactive power capability of the generator and its reactive power generation at the current operating point. This quantity may not represent the useful quantity of the GRPR since at the collapse point all the amount of the TGRPR cannot be utilized. Effective generator RPR (EGRPR), as achievable representative of the GRPR, is defined as the difference between the generator's reactive power output at the voltage collapse point and the generator's reactive power output at the current operating point. The TGRPR is an upper bound for the EGRPR. The LRPR, the TGRPR, and the EGRPR for the two bus test system are shown in Fig. 1c and b.

The system operator defines the set-points of the voltage and reactive power controllers by using different criteria such as minimization of reactive power injection (or maximization of TGRPR), minimization of voltage profile deviation, and minimization of transmission losses. These different objectives would result into different amount of RPR and consequently different security margins. Nevertheless, the RPRs should be appropriately managed from the available resources to enhance the VSM.

Improving the VSM has been considered in the literature in different ways. The proposed VAR scheduling methods in [6-8] add a penalty factor to the OPF to maximize the VSM. The penalty factor is derived from the eigenvectors and/or the generators' participation factors related to the Jacobian matrix.

RPR provision is widely proposed in literature based on: (a) security constrained OPF (SCOPF) to assess the RPR with different constraints $[3,9]$ and (b) voltage stability constrained OPF (VSCOPF) to determine preventive $[10,11]$ and corrective [10] controls considering voltage stability.

Regarding the literatures on $L R P R$ [12], defines a reactive reserve as the sum of the exhausted reactive reserves at the minimum point of the $Q V$-curve. The RPR-based contingency constrained OPF (RCCOPF) presented in [3] utilizes a decomposition method to solve the preventive voltage control in normal state while considering the active power margin of post-contingency states. The proposed RPR management in [5] utilizes a two level Benders decomposition, including a base case and stressed cases, to ensure the feasibility of the stressed cases.

Most of the studies on GRPR like in [13] and [14] are performed on TGRPR since it can be calculated easily regardless stability analysis. On the other hand, EGRPR depends on the generators capability curve and the network characteristics [15]. That means the maximization of TGRPR does not imply necessarily the maximization of EGRPR all the times. The GRPR is studied from the EGRPR point of view more in depth in [15] and [16]. The EGRPR for a bus or an area is determined in [17] as the weighted sum of the individual 
RPR of generators at the minimum of the $Q V$-curve. The proposed approach in [18], determines the minimum RPR to face a contingency, while stressing the system in its pre-contingency state, until reaching an unacceptable post-contingency response.

The proposed two-step approach in [9] determines the required RPR first by finding, using a SCOPF, the minimum overall needed RPR from the generators for postulated contingencies. Then, additional RPR requirements are determined to account for the dynamic system behavior and to ensure the voltage stability of certain contingencies.

Reference [19] investigates the correlative relationship between the GRPR and the system VSMs for on-line monitoring. A nonlinear relationship between the GRPR and the VSMs and the voltage limits violations is investigated in [20].

This classification of RPR is necessary to distinguish the purpose of each study and to avoid mixing different concepts. In this paper, we concentrate on GRPR and specifically the maximization of $E G R P R$ as the main preventive action against voltage instability. This optimization determines the reactive power generation and its reserve for each generator such that maximum voltage stability can be attained for the system. Note that this optimization is performed for a given active power operating point.

This preventive action by increasing the security margin can decrease or even remove the necessity of the corrective action in case of contingency. Furthermore, application of the proposed optimization method can be utilized as the objective of tertiary voltage regulation (TVR), to improve VSMs.

\section{Proposed method for reactive power reserve management}

The management of the reactive power generation and its reserve is a correlated task for ensuring the voltage stability of the system that strongly depends on the generators and transmission system capabilities. The power supplied by a generator is constrained by its capability curve. For a given active power output, the maximum reactive power support of a generator is obtained while considering the limitation of the field current $\left(\bar{Q}_{r i}\right)$, the limitation of the armature current $\left(\bar{Q}_{a i}\right)$ and the under-excitation limit [21]. The under-excitation limit is considered by the inequality constraint $Q_{i}>Q_{i}^{\min }$, where $Q_{i}^{\min }$ is negative and represents the generator minimum reactive power output. The maximum produced reactive power regarding the field and armature limitations is given by (1) and (2), respectively.

$Q_{i}^{\max }=\min \left\{\bar{Q}_{r i}=-\frac{V_{i}^{2}}{X_{s i}}+\sqrt{\frac{V_{i}^{2} \bar{I}_{f i}^{2}}{X_{s i}^{2}}-P_{i}^{2}}\right\}$

$Q_{i}^{\max }=\min \left\{\bar{Q}_{a i}=\sqrt{V_{i}^{2} \cdot \bar{I}_{a i}^{2}-P_{i}^{2}}\right.$

where $i$ is the index of the generators, $V_{i}$ is the generator terminal voltage, $P_{i}$ is the generator active power output, $\bar{I}_{f i}$ is the maximum field current, $\bar{I}_{a i}$ is the maximum armature current, and $X_{s i}$ is the synchronous reactance. $Q_{i}^{\max }$ is defined as the minimum of (1) and (2) to avoid an additional constraint on the upper limit of $Q_{i}$ and also additional variables for complementarity constraints as it is explained in the next paragraph and Section 3.1. These detailed models of the generator operating limits must be considered in order to utilize the maximum reactive power capability and to meet the reactive power demands during emergency states [5].

Moreover, three modes of generator operation, namely within voltage control range, over-excitation and under-excitation should be considered in the evaluation of the VSM and RPR. Over/under excitation is considered when the maximum reactive power limit is reached [9]. The generator switch between the constant terminal voltage and the constant reactive power output is handled by the following complementarity problem:

$0 \leq\left(Q_{i}^{C}-Q_{i}^{\min }\right) \perp V_{i}^{u e} \geq 0$

$0 \leq\left(Q_{i}^{\max }-Q_{i}^{C}\right) \perp V_{i}^{o e} \geq 0$

$V_{i}^{C}=V_{i}^{*}+V_{i}^{u e}-V_{i}^{o e}$

where the operator $\perp$ denotes the complementarity of two quantities. The voltage magnitudes at the collapse point $\left(V_{i}^{C}\right)$ are defined as the sum of the voltage at the operating point $\left(V_{i}^{*}\right)$ plus the underexcitation voltage $\left(V_{i}^{u e}\right)$ and minus the over-excitation voltage $\left(V_{i}^{o e}\right)$ [22]. In this formulation $\left(Q_{i}^{C}\right)$ is the generator reactive power output at the collapse point. $Q_{i}^{\max }$ is the maximum reactive power output obtained from (1) and (2). $Q_{i}^{\min }$ is the minimum reactive power output that represents the under-excitation limit. The complementarity model allows the voltage levels to be changed when generators reach reactive power limits. These complementarity constraints (3) and (4) could be, respectively, taken into consideration by the following nonlinear constraints:

$\left(Q_{i}^{C}-Q_{i}^{\min }\right) \cdot V_{i}^{u e} \leq 0 \quad(i \in N G: P V$ nodes $)$

$\left(Q_{i}^{\max }-Q_{i}^{C}\right) \cdot V_{i}^{o e} \leq 0 \quad(i \in N G: P V$ nodes $)$

In order to prevent a strict complementarity constraint and the related problems [22], the righthand sides' zeros of (6) and (7) are replaced by a small positive number $\left(\varepsilon=10^{-7}\right)$.

For the $i$ th generator, TGRPR and EGRPR are defined by the following equations, as shown in Fig. 1c:

$T_{G R P R_{i}}=Q_{i}^{\max }-Q_{i}$

$E G R P R_{i}=Q_{i}^{C}-Q_{i}$

where $Q_{i}$ is the generator reactive power output at the operating point. Note that in the definition of TGRPR given in (8), the first term, $Q_{i}^{\max }$, depends on the voltage magnitude of the generators.

The objective of the proposed method for the reactive power generation and reserve management is to maximize EGRPR and as a consequence to improve the VSMs at the pilot nodes. The pilot nodes are chosen in such a way that by maintaining their voltages at a given level, the voltages of the whole region buses are kept at a desirable level. As a result, increasing the VSM of the pilot nodes inherently improves the voltage stability of the system.

Therefore, the maximization of the EGRPR, given in (9), can also be formulated as follows. Similar formulation could be presented for the maximization of TGRPR.

$$
\begin{aligned}
\max E G R P R & =\max \sum_{i \in \mathrm{NG}}\left\{Q_{i}^{C}-Q_{i}\right\} \\
& \stackrel{=}{\min } \sum_{i \in \mathrm{NG}}\left\{Q_{i}-Q_{i}^{C}\right\}
\end{aligned}
$$

This optimization consists in the minimization of the difference between the sum of the generators reactive power output at the operating $\left(\Sigma Q_{i}\right)$ and the collapse point $\left(\Sigma Q_{i}^{C}\right)$. The vector of the control variables $(u)$ includes the voltage of $P V$ generators and the reactive power output of $P Q$ generators. The control variables could be considered as the complicating variables since they are present in the current operating point and the voltage collapse point. Therefore, this optimization problem could be solved according to two different ways: one-stage (simultaneous) or two-stage [22]. In this paper, these two optimization approaches are investigated to solve the proposed optimization.

The overall structures and general formulation of the two approaches are illustrated in Fig. 2. In the presented general formulation $f\left(x, x^{C}, u, s\right)$ is maximization of EGRPR. $x$ indicates the vector 


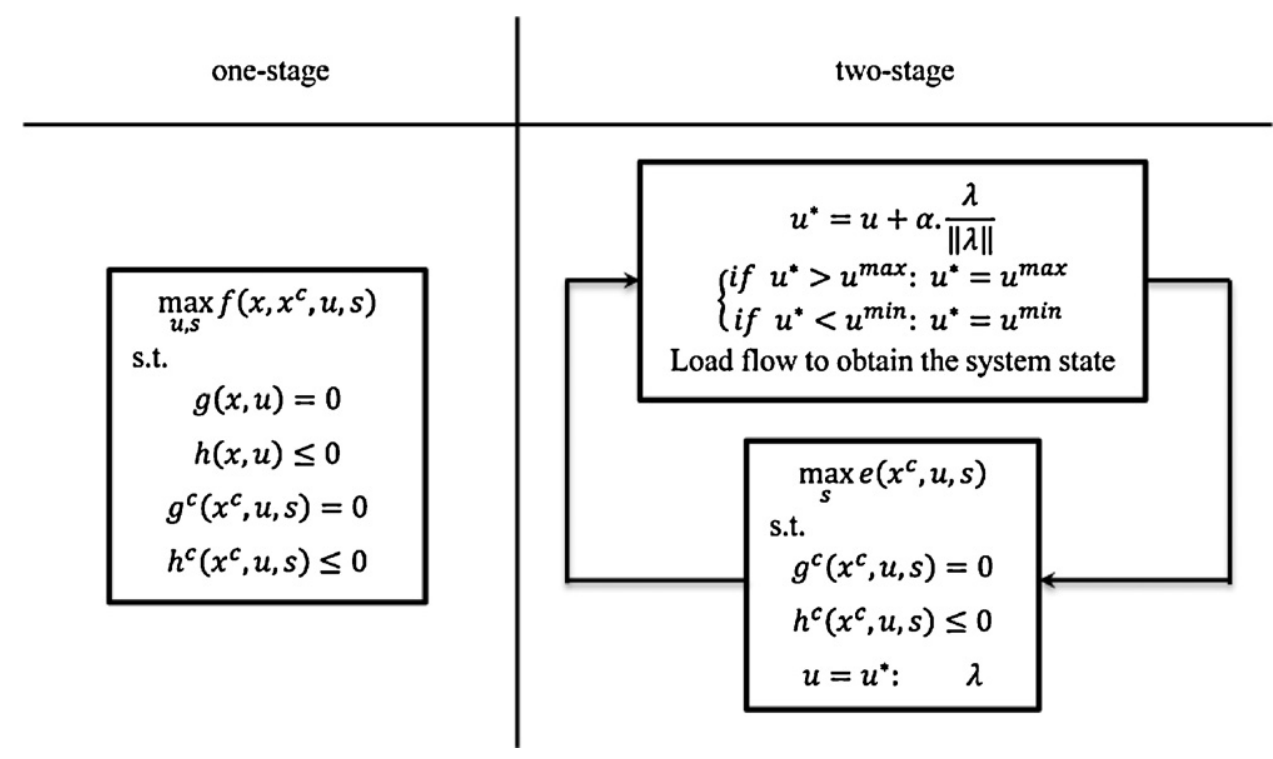

Fig. 2. Structure of one-stage and two-stage optimization.

of the state variables and $s$ is the vector of injected fictitious reactive power for pilot nodes at the voltage collapse point. $g(x, u)$ and $h(x, u)$ correspond to equality and inequality constraints, respectively. Superscript " $C$ " represents the variables at the collapse point. Additionally for two-stage optimization, $e\left(x^{C}, u, s\right)$ is the maximization of the generators reactive power output at the collapse point $\left(\Sigma Q_{i}^{C}\right) \cdot u^{*}$ stands for the values of the control variables which are used as the input for this optimization.

Before explaining the details of these two approaches, it is worth to mention that the proposed optimization looks like similar to the traditional voltage and reactive power management based on the maximum loading margin [22], since both of them consider the analysis of a current operating point and a collapse point. However, the specifications of the objective function and the collapse point for the maximization of the loading margin and the EGRPR are quite different. The collapse point for the maximization of the loading margin is obtained by increasing the loading level of the whole system linearly in one direction until reaching a bifurcation point whereas the collapse point in case of EGRPR maximization is attained based on the reactive power margins at the pilot nodes.

In comparison to the contingency constrained optimization methods [(3),(5) and (9)], the proposed approach takes into account only one voltage emergency state that is the collapse point. Therefore, the proposed method does not need any contingency selection or analysis of several contingencies. However, the proposed optimization could be developed to provide appropriate EGRPR for a set of postulated contingencies.

\subsection{Simultaneous optimization of EGRPR for current operating and collapse points}

This approach assumes that the state variables at both of the current operating point and the voltage collapse point are the optimization variables in addition to the control variables. Hence, the relationship between the current operating point and the voltage collapse point is taken into consideration. Therefore, the maximization of EGRPR given by (10) is subjected to the following equality and inequality constraints:

- at the operating point (11)-(17):
$P_{i n}-P_{d n}+\sum_{m \in N B} V_{n} V_{m}\left(G_{n m} \cos \theta_{n m}+B_{n m} \sin \theta_{n m}\right)=0, \quad n \in N B$

$Q_{i n}-Q_{d n}+\sum_{m \in N B} V_{n} V_{m}\left(G_{n m} \sin \theta_{n m}-B_{n m} \cos \theta_{n m}\right)=0, \quad n \in N B$

$\left(G_{n m}^{2}+B_{n m}^{2}\right) \cdot\left(\left(V_{n}\right)^{2}+\left(V_{m}\right)^{2}-2 V_{n} V_{m} \cos \theta_{n m}\right) \leq\left(I_{l}^{\max }\right)^{2}$,

$\{n, m\} \in l, l \in N L$

$Q_{i}^{\min } \leq Q_{i} \leq Q_{i}^{\max }, \quad n \in N B$

$V_{n}^{\min } \leq V_{n} \leq V_{n}^{\max }, \quad n \in N B$

- and at the voltage collapse point (16)-(24):

$P_{i n}^{C}-P_{d n}+\sum_{m \in N B} V_{n}^{C} V_{m}^{C}\left(G_{n m} \cos \theta_{n m}^{C}+B_{n m} \sin \theta_{n m}^{C}\right)=0, \quad n \in N B$

$Q_{i n}^{C}-Q_{d n}-Q_{f p}+\sum_{m \in N B} V_{n}^{C} V_{m}^{C}\left(G_{n m} \sin \theta_{n m}^{C}-B_{n m} \cos \theta_{n m}^{C}\right)=0$,

$p \in N P, n \in N B$

$\left(G_{n m}^{2}+B_{n m}^{2}\right) \cdot\left(\left(V_{n}^{C}\right)^{2}+\left(V_{m}^{C}\right)^{2}-2 V_{n}^{C} V_{m}^{C} \cos \theta_{n m}^{C}\right) \leq\left(I_{l}^{\max }\right)^{2}$,

$\{n, m\} \in l, l \in N L$

$V_{n}^{\min } \leq V_{n}^{C} \leq V_{n}^{\max }, \quad n \in N B-(n \in N G: P V$ nodes $)$

$V_{i}^{C}=V_{i}^{*}-V_{i}^{o e} \quad(i \in N G: P V$ nodes $)$

$\left(Q_{i}^{\max }-Q_{i}^{C}\right) \cdot V_{i}^{o e} \leq 0 \quad(i \in N G: P V$ nodes $)$

$V_{i}^{o e} \geq 0 \quad(i \in N G: P V$ nodes $)$ 
$Q_{i}^{\min } \leq Q_{i}^{C} \leq Q_{i}^{\max } \quad(i \in N G)$

$Q_{i}^{C}=Q_{i}^{*} \quad(i \in N G: P Q$ nodes $)$

where the variables without and with superscript $C$ represent the variables related to the operating point and the collapse point, respectively. In this formulation $N B, N L, N G$ and $N P$ are the number of buses, the number of lines, the number of generators, and the number of pilot nodes, respectively. $n$ and $m$ are the index of buses, $l$ is the index of lines, $i$ is the index of generators, $d$ is the index of demands and $p$ is the index of pilot nodes. $V_{n}$ is the voltage magnitude of bus $n$, and $\theta_{n m}$ is the voltage angle difference between the buses $n$ and $m . G_{n m}$ and $B_{n m}$ are the real and imaginary part of the $\{n, m\}$ element of the admittance matrices. The active power and the reactive power are shown by $P$ and $Q$ respectively. The reactive power capacity limits of each generator are specified by $Q_{i}^{\min }$ and $Q_{i}^{\max }$. The limits of voltages at bus $n$ are $V_{n}^{\min }$ and $V_{n}^{\max }$. The maximum transfer capability of the transmission lines is given by $I_{l}^{\max }$.

At the current operating point (resp. voltage collapse point) the active and reactive power balance equality constraints are given by (11) and (12) (resp. (16) and (17)). Note that the reactive power balance equality constraint given by (17) is different from (12). In (17) the variable $Q_{f p}$ is added that demonstrates the fictitious reactive power injection (load) at the pilot nodes. The positive and negative values of $Q_{f p}$ are devoted to the reactive power consumption and generation, respectively. The transmission lines flow limit is considered in (13) (resp. (18)). The limits of the generators reactive power and the voltage magnitude at each bus are considered in (14) and (15), respectively. Note that the generators limits are considered with their capability curves obtained from (1) and (2). At the voltage collapse point, the limitations of the generators reactive power output and the voltage of the buses are given by (19)-(24).

$V_{i}^{*}$ and $Q_{i}^{*}$ in (20) and (24) are the voltage and reactive power of the PV and PQ generators at the operating point, respectively. These two equality constraints in addition to the inequality constraints (21) and (22) correlate the current operating point and the collapse point while considering the complementarity constraints mentioned by (5)-(7). It should be noted that at the collapse point, the generators switching to the under-excited mode is not taken into consideration. In fact, in response to the increase of the fictitious reactive power loads at the pilot nodes, the generators need rather to switch from the voltage control mode to the over-excited mode in order to increase their reactive power support at the collapse point. Besides the fact that the PQ generators reactive power output are the same at the current operating point and at the voltage collapse point (according to (24)), they can participate in the optimization process.

\subsection{Two-stage optimization for the current operating point and the collapse point}

The maximization of EGRPR given by (10) can be solved in two stages through decomposing the problem into two smaller problems. The first stage determines an operating point and the second stage calculates the collapse point based on the results of the first stage. These two smaller problems are generally solved much simpler than a single larger one [22]. However, a drawback of this approach is that the solution of the two-stage optimization is obtained iteratively. Several ways can be proposed to correlate these two stages and to fix the operating point [22].

Ref. [16] decomposes the problem in (10) using the Bender's decomposition method with a master problem (operating point) and a sub problem (collapse point). The proposed method in [16] does not consider the generators switch between constant terminal voltage and constant reactive power output. The
Benders' decomposition provides acceptable results in the absence of the complementarity constraints (5)-(7) as demonstrated in [16]. However, it is not effective whenever these constraints are taken into considerations. Here, to overcome this problem a Subgradient method is used as a bi-level optimization method.

Subgradient methods are iterative first-order methods which can be applied to a variety of problems [23]. They are simple to implement and their computational burden is small. However, their progress to the optimum is slow and oscillatory [24]. In this paper, a subgradient method iteratively updates the control variables as follow (see Fig. 2):

$u^{(k+1)}=u^{(k)}+\alpha_{k} \cdot \frac{\lambda^{(k)}}{\left\|\lambda^{(k)}\right\|}$

Here $u^{(k)}$ is the vector of the control variable at $k$ th iteration, $\lambda^{(k)}$ is the subgradient of the objective function $\left(e^{(k)}\right)$ with respect to $u^{(k)}$, and $\alpha_{k}>0$ is the $k$ th step size. $\left\|\lambda^{(k)}\right\|$ is the Euclidean norm of $\lambda^{(k)}$ which normalize the magnitude of the subgradients for the set of PV and PQ generators separately. The subgradients $\lambda^{(k)}$ are obtained from the solution of the optimization at the voltage collapse point (sub-problem). For the voltage at PV nodes and the reactive power at PQ nodes (the control variables) $\lambda^{(k)}$ components are the Lagrangian multipliers of (20) and (24), respectively. Many different types of step size schemes can be used [23]. In this paper, the so called non summable but square summable step size is selected. It is given as follows:

$\alpha_{k}=\frac{a}{b+k}$

where $a$ and $b$ are positive constant scalars. Here, $1.5 \leq a \leq 2.5$ and $b=20$. They should be chosen for each specific problem. Note that whenever the obtained control variables in (25) in $k$ th iteration go beyond their upper or lower limits, their values are set at the corresponding limits as shown in Fig. 2.

Then, a power flow is performed to evaluate the EGRPR in each iteration $k$ and to determine, respectively, the voltage and reactive power at PQ and PV generators. If the power flow solution demonstrates that voltages of PQ generators are beyond the limits, the corresponding control variables are iteratively reduced by $2.5 \%$. This procedure iterates till obtaining the state variables (voltage magnitudes and angles) within allowed limits.

Since the subgradient method is not a descent (resp. ascent) method in minimization (resp. maximization), the best objective value found so far $\left(f_{\text {best }}^{(k)}\right)$ should be kept for tracking the optimum.

$f_{\text {best }}^{(k)}=\max \left\{f\left(u^{(1)}\right), \ldots, f\left(u^{(k)}\right)\right\}$

The algorithm converges when $\lim _{k \rightarrow \infty} f\left(u^{(k)}\right)-f^{*} \leq \delta$ where $f^{*}$ denotes the optimum solution of the problem obtained from one-stage approach and $\delta$ is a small number. More sophisticated stopping criteria are presented in [23].

In the two-stage optimization, the appropriate selection of the starting point $\left(u^{(1)}\right)$ is an important subject. The initial voltage set points equal to 1 is not always an appropriate starting point. The authors found that, the results of the TGRPR maximization problem, given by (8), is an appropriate starting point; because its result leads to wider feasible region in the EGRPR maximization problem.

\subsection{Pilot node selection}

The pilot nodes are the most voltage sensitive nodes that reflect the state of the voltage in a control zone. The optimal selection of the pilot nodes for a zonal or a secondary voltage control is studied in different literatures [25]. They could be selected for a set of contingencies [25] or random reactive power disturbances [26,27]. In this paper, the term pilot node is explicitly used for the buses 
Table 1

The 6-bus system generators data.

\begin{tabular}{|c|c|c|c|c|c|c|}
\hline & \multirow[t]{2}{*}{ Bus } & \multirow[t]{2}{*}{$P_{g}^{0}(\mathrm{MW})$} & \multirow[t]{2}{*}{$P_{g}^{\max }(\mathrm{MW})$} & \multicolumn{3}{|c|}{ Capability curve } \\
\hline & & & & $\bar{I}_{f}(\mathrm{pu})$ & $\bar{I}_{a}(\mathrm{pu})$ & $X_{S}(\mathrm{pu})$ \\
\hline Gen 1 & 1 & - & 200 & 1.37 & 2.20 & 0.20 \\
\hline Gen 2 & 2 & 50 & 150 & 1.35 & 1.65 & 0.25 \\
\hline Gen 3 & 3 & 60 & 180 & 1.36 & 1.98 & 0.23 \\
\hline
\end{tabular}

where the fictitious reactive power injections $\left(Q_{f}\right)$ are connected to. It is assumed that these pilot nodes are the most voltage sensitive nodes.

\section{Test case study}

The proposed method for the maximization of EGRPR is tested on 6-bus system for two different loading levels to demonstrate the evolution of EGRPR of each generator. The simulations are carried out for the case with PV generators only and the case including both PV and PQ generators. Then, the mechanism according which each generator increases its participation in EGRPR is described. In addition, the effectiveness of the proposed methods in the case of a larger power system is investigated using the New England 39-bus system. For each case study, the optimization is performed with three different approaches listed below and the results are compared.

(1) minimization of the generated reactive power (min. Q)

(2) maximization of the EGRPR in one-stage (max. EGRPR Os)

(3) maximization of the EGRPR in two-stage (max. EGRPR Ts)

The first approach is taken into consideration as the reference case that aims at minimizing the total generated reactive power. This objective could be interpreted as the minimization of the reactive power cost. The last two approaches (max. EGRPR), as described in Section 3, have the same objective.

The OPF model is a nonlinear problem which is solved using "fmincon" with interior-point algorithm [28] in MATLAB R2011a.

\subsection{6-Bus study case}

The 6-bus test system is shown in Fig. 3. The system contains 3 generators, 3 loads, and 11 transmission lines. The data for the 6 -bus system are provided in Tables $1-3$. Table 1 shows the generators' data including the current active power dispatch and the capability curves according to (1) and (2). The buses data are given

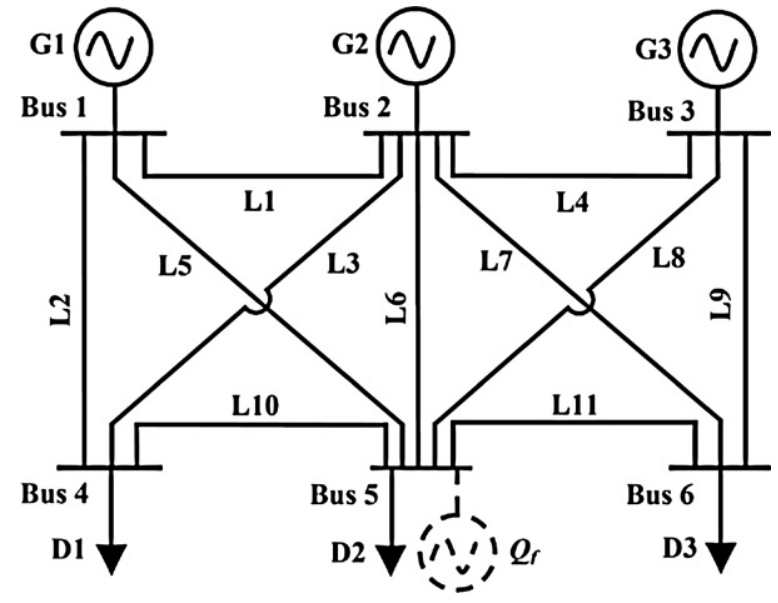

Fig. 3. One line diagram of 6-bus system. in Table 2, including each bus type and its active and reactive power load. Table 3 provides the branches data, including the two ends bus numbers, the series resistances $(R)$, the series inductances $\left(X_{L}\right)$, the shunt conductance $\left(X_{G}\right)$, and the maximum transfer capabilities $\left(I^{\max }\right)$. The voltage deviation of all buses is acceptable within $\pm 10 \%$ of the nominal voltage. In this study case, bus 5 is selected as the pilot node since it is directly connected to all generators.

In one simulation, all generators are assumed as PV generators (G1 is the swing generator and G2 and G3 are PV generators) and in another one, G3 is considered as PQ generator. For the swing generator, $P_{\mathrm{G} 1}$ is considered as an unknown variable to compensate the active power losses. The change of the active power losses is due to different scheduling of voltage and reactive power resources and thus different reactive power flows. The simulation is performed for two load level; the load level mentioned in Table 1 (low loading) and a $20 \%$ increase of that load (high loading).

The simulation results with different approaches for the high (low) loading level are shown in Fig. 4 and Tables 4 and 5 (Fig. 5 and Tables 6 and 7). In Tables 4-7, the obtained solutions for the control variables are given in bold.

Figs. 4 and 5 illustrate the sum of all generators EGRPR for the 3 different approaches for high and low loading levels, respectively. The horizontal axes are devoted to the number of iterations, since the two-stage approach is iterative. The comparison of Figs. 4 and 5 shows that, the EGRPR is lower for high loading level than for low loading level for all approaches. Likewise, the EGRPR of the twostage approach approximately converges to the amount of the onestage approach. The maximum difference between their solutions is lower than $1.2 \%$.

Besides, in the case with PV generators only (3 PV generators), the amounts of EGRPR for the three approaches are close to each

Table 2

The 6-bus system bus data.

\begin{tabular}{llcc}
\hline Bus & Type $^{\mathrm{a}}$ & $P_{d}(\mathrm{MW})$ & $Q_{d}$ (MVAR) \\
\hline 1 & 0 & 0 & 0 \\
2 & 1 & 0 & 0 \\
3 & 1 & 0 & 0 \\
4 & 2 & 70 & 70 \\
5 & 3 & 70 & 70 \\
6 & 2 & 70 & 70 \\
\hline a The numbers stand for the bus types are as follow: 0 for swing node, 1 for PV
\end{tabular}
node, 2 for PQ node, and 3 for Pilot node.

Table 3

The 6-bus system branch data.

\begin{tabular}{llllll}
\hline From bus & to bus & $R(\mathrm{pu})$ & $X_{L}(\mathrm{pu})$ & $X_{G}(\mathrm{pu})$ & $I^{\max }(\mathrm{MVA})$ \\
\hline 1 & 2 & 0.10 & 0.20 & 0.04 & 60 \\
1 & 4 & 0.05 & 0.20 & 0.04 & 90 \\
1 & 5 & 0.08 & 0.30 & 0.06 & 60 \\
2 & 3 & 0.05 & 0.25 & 0.06 & 60 \\
2 & 4 & 0.05 & 0.10 & 0.02 & 90 \\
2 & 5 & 0.10 & 0.30 & 0.04 & 45 \\
2 & 6 & 0.07 & 0.20 & 0.05 & 135 \\
3 & 5 & 0.12 & 0.26 & 0.05 & 105 \\
3 & 6 & 0.02 & 0.10 & 0.02 & 120 \\
4 & 5 & 0.20 & 0.40 & 0.08 & 30 \\
5 & 6 & 0.10 & 0.30 & 0.06 & 60
\end{tabular}


Table 4

Optimization results of 6-bus system for high loading level with 3 PV generators.

\begin{tabular}{|c|c|c|c|c|c|}
\hline Objective & & $G_{1}$ & G2 & G3 & Total \\
\hline \multirow[t]{5}{*}{$\operatorname{Min} Q$} & $V_{i}(\mathrm{pu})$ & 1.1000 & 1.0960 & 1.1000 & - \\
\hline & $V_{i}^{o e}(\mathrm{pu})$ & 0.0000 & 0.0250 & 0.0197 & - \\
\hline & $Q_{i}(\mathrm{MVAR})$ & 29.75 & 108.31 & 88.09 & 226.16 \\
\hline & $Q_{i}^{C}($ MVAR $)$ & 83.54 & 116.42 & 127.31 & 327.27 \\
\hline & EGRPR (MVAR) & 53.78 & 8.11 & 39.22 & 101.11 \\
\hline \multirow[t]{5}{*}{ Max EGRPR one stage } & $V i(p u)$ & 1.0933 & 1.0928 & 1.1000 & - \\
\hline & $V_{i}^{o e}(\mathrm{pu})$ & 0.0000 & 0.0270 & 0.0240 & - \\
\hline & $Q_{i}^{l}($ MVAR $)$ & 25.12 & 109.36 & 92.54 & 227.02 \\
\hline & $Q_{i}^{C}(\mathrm{MVAR})$ & 81.40 & 117.99 & 128.77 & 328.18 \\
\hline & EGRPR (MVAR) & 56.281 & 8.64 & 36.24 & 101.16 \\
\hline \multirow[t]{5}{*}{ Max EGRPR two stages } & $V_{i}(\mathrm{pu})$ & 1.0917 & 1.1000 & 1.0977 & - \\
\hline & $V_{i}^{o e}(\mathrm{pu})$ & 0.0000 & 0.0353 & 0.0227 & - \\
\hline & $Q_{i}^{l}($ MVAR) & 16.21 & 127.75 & 83.02 & 226.98 \\
\hline & $Q_{i}^{C}(\mathrm{MVAR})$ & 80.90 & 118.37 & 129.12 & 328.39 \\
\hline & EGRPR (MVAR) & 64.69 & -9.38 & 46.10 & 101.41 \\
\hline
\end{tabular}

The obtained solutions for the control variables are given in bold.

Table 5

Optimization results of 6-bus system for high loading level with 2 PV and 1 PQ generators.

\begin{tabular}{|c|c|c|c|c|c|}
\hline Objective & & $G_{1}$ & G2 & G3 & Total \\
\hline \multirow[t]{5}{*}{$\operatorname{Min} Q$} & $V_{i}(\mathrm{pu})$ & 1.1000 & 1.0960 & 1.1000 & - \\
\hline & $V_{i}^{o e}(\mathrm{pu})$ & 0.0000 & 0.0347 & - & - \\
\hline & $Q_{i}^{l}(\mathrm{MVAR})$ & 29.75 & 108.31 & 88.09 & 226.16 \\
\hline & $Q_{i}^{C}($ MVAR $)$ & 92.66 & 119.42 & 88.09 & 300.18 \\
\hline & EGRPR (MVAR) & 62.91 & 11.11 & - & 74.02 \\
\hline \multirow[t]{5}{*}{ Max EGRPR one stage } & $V_{i}(\mathrm{pu})$ & 1.0948 & 1.0651 & 1.1000 & - \\
\hline & $V_{i}^{o e}(\mathrm{pu})$ & 0.0000 & 0.0000 & - & - \\
\hline & $Q_{i}^{l}(\mathrm{MVAR})$ & 55.72 & 54.02 & 120.35 & 230.09 \\
\hline & $Q_{i}^{C}($ MVAR $)$ & 83.78 & 118.24 & 120.35 & 322.37 \\
\hline & EGRPR (MVAR) & 28.06 & 64.22 & - & 92.28 \\
\hline \multirow[t]{5}{*}{ Max EGRPR two stages } & $V_{i}(\mathrm{pu})$ & 1.0840 & 1.0673 & 1.0999 & - \\
\hline & $V_{i}^{o e}(\mathrm{pu})$ & 0.0000 & 0.0110 & - & - \\
\hline & $Q_{i}^{l}($ MVAR $)$ & 40.61 & 69.79 & 120.02 & 230.42 \\
\hline & $Q_{i}^{C}($ MVAR $)$ & 80.90 & 120.93 & 120.02 & 321.85 \\
\hline & EGRPR (MVAR) & 40.29 & 51.14 & - & 91.43 \\
\hline
\end{tabular}

The obtained solutions for the control variables are given in bold.

other. The reason is that the generators' switch between the different operation modes (given by (5)-(7)) allows them to change their voltages in order to increase their reactive power output at the collapse point. If the generator mode switch at the collapse point is not taken into consideration, as in the case in [16], the EGRPR becomes larger for max EGRPR than min Q. As shown in Tables 4 and 6, this issue is more evident for the high loading level than for the low loading level because the system reaches its limits.
Furthermore, in the case with PQ generator, although the generator switch mode is considered, max EGRPR noticeably increase the amount of EGRPR in comparison to min $Q$. It is due to the fact that min $Q$ aims to minimize the sum of the generators reactive power outputs at the operating point and this value for the PQ generators is kept constant for the collapse point according to (23). But for max EGRPR, the reactive power outputs of the PQ generators are adjusted in such a way it allows the PV generators

Table 6

Optimization results of 6-bus system for low loading level with 3 PV generators.

\begin{tabular}{|c|c|c|c|c|c|}
\hline Objective & & G1 & G2 & G3 & Total \\
\hline \multirow[t]{5}{*}{$\operatorname{Min} Q$} & $V_{i}(\mathrm{pu})$ & 1.1000 & 1.1000 & 1.1000 & - \\
\hline & $V_{i}^{o e}(\mathrm{pu})$ & 0.0000 & 0.0185 & 0.0085 & - \\
\hline & $Q_{i}(\mathrm{MVAR})$ & 17.32 & 89.65 & 64.94 & 171.91 \\
\hline & $Q_{i}^{C}(\mathrm{MVAR})$ & 71.46 & 113.99 & 124.63 & 310.09 \\
\hline & EGRPR (MVAR) & 54.14 & 24.35 & 59.69 & 138.18 \\
\hline \multirow[t]{5}{*}{ Max EGRPR one stage } & $V_{i}(\mathrm{pu})$ & 1.0941 & 1.0613 & 1.0761 & - \\
\hline & $V_{i}^{o e}(\mathrm{pu})$ & 0.0000 & 0.0000 & 0.0000 & - \\
\hline & $Q_{i}(\mathrm{MVAR})$ & 54.69 & 44.06 & 77.55 & 176.31 \\
\hline & $Q_{i}^{C}(\mathrm{MVAR})$ & 89.39 & 97.65 & 129.99 & 317.03 \\
\hline & EGRPR (MVAR) & 34.69 & 53.59 & 52.44 & 140.72 \\
\hline \multirow[t]{5}{*}{ Max EGRPR two stages } & $V_{i}(\mathrm{pu})$ & 1.1000 & 1.0663 & 1.1000 & - \\
\hline & $V_{i}^{o e}(\mathrm{pu})$ & 0.0000 & 0.0000 & 0.0202 & - \\
\hline & $Q_{i}(\mathrm{MVAR})$ & 52.23 & 24.52 & 98.73 & 175.48 \\
\hline & $Q_{i}^{C}($ MVAR $)$ & 91.01 & 98.13 & 128.71 & 317.84 \\
\hline & EGRPR (MVAR) & 38.78 & 73.61 & 29.98 & 142.37 \\
\hline
\end{tabular}

The obtained solutions for the control variables are given in bold. 
Table 7

Optimization results of 6-bus system for low loading level with 2 PV and 1 PQ generators.

\begin{tabular}{|c|c|c|c|c|c|}
\hline Objective & & G1 & G2 & G3 & Total \\
\hline \multirow[t]{5}{*}{$\operatorname{Min} Q$} & $V_{i}(\mathrm{pu})$ & 1.1000 & 1.1000 & 1.1000 & - \\
\hline & $V_{i}^{o e}(\mathrm{pu})$ & 0.0000 & 0.0398 & - & - \\
\hline & $Q_{i}(\mathrm{MVAR})$ & 17.32 & 89.65 & 64.94 & 171.91 \\
\hline & $Q_{i}^{C}(\mathrm{MVAR})$ & 97.35 & 120.71 & 64.94 & 283.00 \\
\hline & EGRPR (MVAR) & 80.03 & 31.07 & - & 111.10 \\
\hline \multirow[t]{5}{*}{ Max EGRPR one stage } & $V_{i}(\mathrm{pu})$ & 1.0801 & 1.0472 & 1.1000 & - \\
\hline & $V_{i}^{o e}(\mathrm{pu})$ & 0.0000 & 0.0000 & - & - \\
\hline & $Q_{i}($ MVAR $)$ & 48.0466 & 9.86 & 121.57 & 179.48 \\
\hline & $Q_{i}^{C}(\mathrm{MVAR})$ & 88.72 & 101.80 & 121.57 & 312.09 \\
\hline & EGRPR (MVAR) & 40.67 & 91.94 & - & 132.61 \\
\hline \multirow[t]{5}{*}{ Max EGRPR two stages } & $V_{i}(\mathrm{pu})$ & 1.0708 & 1.0414 & 1.1000 & - \\
\hline & $V_{i}^{o e}(\mathrm{pu})$ & 0.0000 & 0.0000 & - & - \\
\hline & $Q_{i}(\mathrm{MVAR})$ & 43.05 & 8.82 & 129.11 & 180.98 \\
\hline & $Q_{i}^{C}(\mathrm{MVAR})$ & 83.33 & 100.06 & 129.11 & 312.50 \\
\hline & EGRPR (MVAR) & 40.29 & 91.24 & - & 131.53 \\
\hline
\end{tabular}

The obtained solutions for the control variables are given in bold.

Table 8

VSM and corrective actions of the given contingencies for 6-bus system for high loading with 2 PV and 1 PQ generators.

\begin{tabular}{|c|c|c|c|c|}
\hline \multirow[t]{2}{*}{ Contingency } & & \multirow[t]{2}{*}{ VSM (MVAR) } & \multicolumn{2}{|l|}{ Corrective actions } \\
\hline & & & $\begin{array}{l}\text { Voltage and reactive power } \\
\text { rescheduling }\end{array}$ & $\begin{array}{l}\text { Active power rescheduling } \\
\text { or load shedding }\end{array}$ \\
\hline \multirow[t]{2}{*}{ L1 } & $\operatorname{Min} Q$ & -78.22 & - & - \\
\hline & Max EGRPR & -123.78 & - & - \\
\hline \multirow[t]{2}{*}{ L3 } & $\operatorname{Min} Q$ & 51.53 & $\Delta V_{2}=-0.0348(\mathrm{pu}) \Delta Q_{3}=12.32(\mathrm{MVAR})$ & - \\
\hline & Max EGRPR & -59.20 & - & - \\
\hline \multirow[t]{2}{*}{ L8 } & $\operatorname{Min} Q$ & - & - & $9.59(\mathrm{MW})$ \\
\hline & Max EGRPR & - & - & $9.59(\mathrm{MW})$ \\
\hline
\end{tabular}

${ }^{\text {a }}$ Here, load shedding is the case and it is executed with constant power factor.

to increase their EGRPR. As shown in Table 5 (resp. in Table 7), these results demonstrate 17.83 MVAR (20.97 MVAR) increase in EGRPR, and 21.93 MVAR (29.29 MVAR) increase in the generators reactive power outputs at the voltage collapse point, with only 4.1 MVAR (8.32 MVAR) increase in generation reactive power at the current operating point. The reactive power output of G3 increases from 88.09 MVAR (64.94 MVAR) to 120.35 MVAR(121.57 MVAR), to reduce the required reactive power of other generators at the current operating point. It helps G1 and G2 to decrease their reactive power generation at the current operating point, and to improve their reactive power output at the voltage collapse point, and consequently increase their EGRPRs, as shown in Table 5 (Table 7). Comparing the cases with three and two PV generators, the system
EGRPR decreases from 101.28 MVAR(141.54 MVAR) to 91.85 MVAR (132.068 MVAR) because the number of voltage control generators is lower.

In the optimization process, the EGRPR of each generator increases by different ways as depicted in Fig. 6a-c. In these figures, the EGRPR for min $Q$ and $\max E G R P R$ are given by $E G R P R_{i}^{0}$ and $E G R P R_{i}^{*}$, respectively. In the first case, as shown in Fig. 6a, a small increase of $Q_{i}$, leads to a higher increase of $Q_{i}^{C}$. In the second case, as shown in Fig. 6b, with a large decrease of $Q_{i}, Q_{i}^{C}$ decreases slightly. Finally in Fig. $6 c$, since $Q_{i}^{C}$ reaches the maximum capability of the generator $\left(Q_{i}^{\max }\right)$ it cannot go further, so in this case, EGRPR increases by reducing $Q_{i}$.

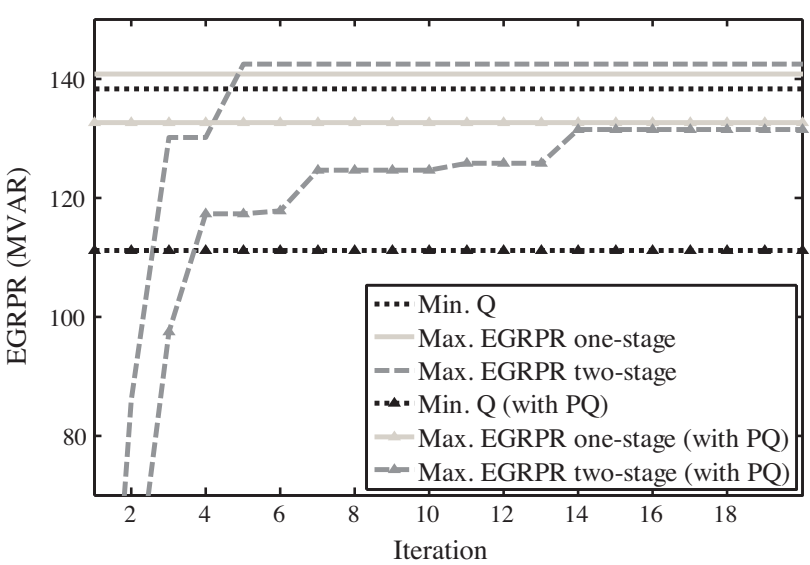

Fig. 5. EGRPR of 6-bus system in low loading.
Fig. 4. EGRPR of 6-bus system in high loading.

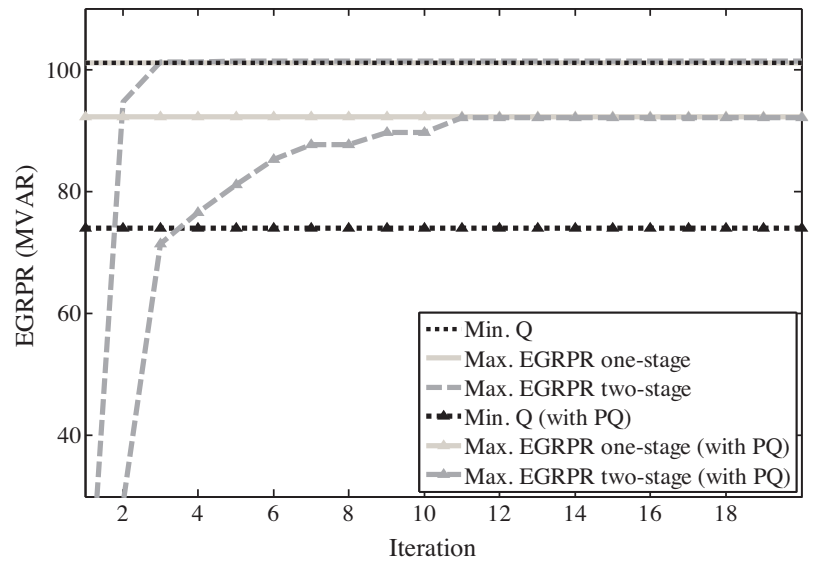



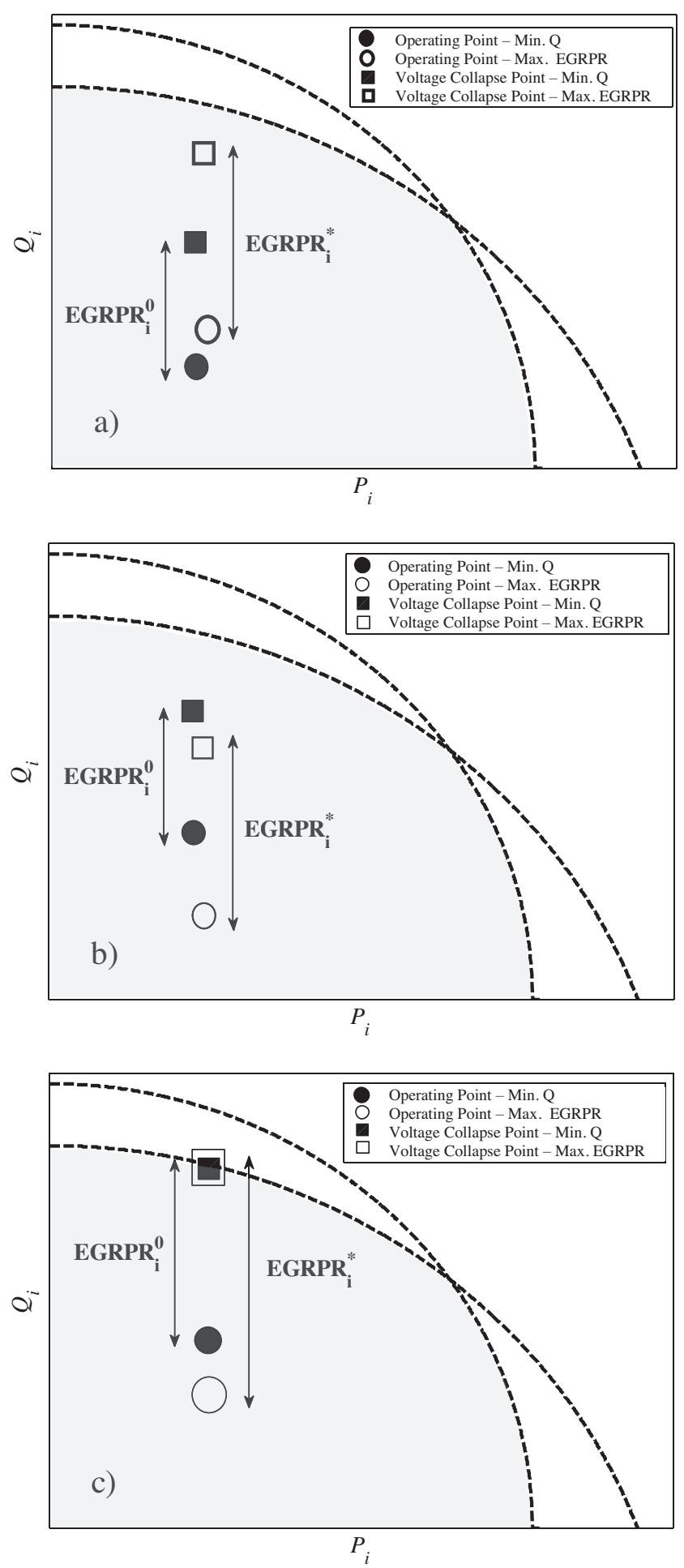

Fig. 6. Increase of EGRPR by different ways: (a) low increase of $Q_{i}$ with higher increase of $Q C_{i}$, (b) big descend of $Q_{i}$ with lower decrease of $Q C_{i}$, and (c) drop in $Q_{i}$ while $Q C_{i}$ is reached to the generator's maximum output $\left(Q_{i}^{\max }\right)$.

The effectiveness of the proposed optimization methods regarding the transmission system contingencies is investigated in the case of 6-bus system with 2 PV and 1 PQ generators for the high loading level. As shown in Table 8, three contingencies are selected to illustrate three different types of post-contingency states. The contingencies are studied in two cases, min $Q$ and max EGRPR Os. For each contingency, the VSM as well as the type and the amount of the corrective actions for unstable cases are given. In this respect, the VSM is assumed to be the sum of the LRPR for all load buses. For every load bus an OPF is solved to calculate VSM from QV-curve. The negative (resp. positive) values of the VSM indicate the margin to instability (resp. stability). Note that the voltage and the reactive power rescheduling, as well as the active power rescheduling and the load shedding are considered as corrective actions, respectively.

In the case of contingency L1, the system is stable for min $Q$ and max EGRPR, but the VSM is much higher in the latter. For contingency L3, the system is unstable for min $Q$ while it remains stable in max EGRPR. For min $Q$, the required corrective action was the voltage and reactive power rescheduling. The proposed optimization decreases the number of such unstable contingencies and consequently the necessity of corrective actions. This situation is more observed for the high loading level. For contingency L8, the system is unstable in min $Q$ and max EGRPR since the OPFs for obtaining the VSMs do not converge. Indeed, the voltage and reactive power rescheduling is not effective and corrective actions such as the active power rescheduling or load shedding should be taken. Thus, the amount of the corrective action (here load shedding) is the same in min Q and max EGRPR. It is worth to mention that different OPFs are developed to calculate the corrective actions.

\subsection{New England 39-bus system}

The effectiveness of the proposed method is demonstrated as well using New England 39-bus system as depicted in Fig. 7. The data of the generators for the New England 39-bus system is provided in Table 9 . The data of the buses and the branches can be found in literature. The voltage of each bus is acceptable between 0.94 and $1.06 \mathrm{pu}$ In this simulation, G6 and G8 are assumed as PQ generators.

As it is mentioned in Section 3.3 the results of the proposed method depends on the selection of the pilot nodes. Thus, buses 4 , $7,8,12,20,21,23,24,25$ and 28 are selected as the most voltage sensitive buses based on the presented results in [27].

The simulation results for New England 39-bus system are given in Figs. 8-10. The system EGRPR increases by the proposed oneand two-stage reactive power scheduling as illustrated in Fig. 8. The 18.07 MVAR increase in the reactive power output of generators is the additional cost for gaining 86.06 MVAR increase in the generator's reactive power support at the voltage collapse point. The share of each generator's in the system EGRPR by different optimization approaches is shown in Fig. 9. The generators reactive power outputs at the current operating point and the voltage collapse point with different optimization approaches is demonstrated in Fig. 10. The EGRPRs of G1 and G5 significantly increase, since they are the most important generators for the voltage and reactive power control. On the other hand, the EGRPRs of G4, G9, and G10 decrease because their RPRs are not effective for the voltage and reactive power control. Also the EGRPRs of G2, G3, and G7 remain approximately unchanged. Note that the EGRPR of G6 and G8, as PQ generators, is equal to zero. As illustrated in Fig. 10, they play a role in the optimization by increasing their reactive power output.

As Fig. 10 shows, the reactive power output of all generators at the operating point increase, except G1 and G5. These generators reactive power outputs at the operating point are reduced significantly, while their reactive power outputs at the voltage collapse point remain unchanged, and as a result, the support of G1 and G5 for voltage control $\left(E G R P R_{1}\right.$ and $\left.E G R P R_{5}\right)$ drastically increases. Moreover, as shown in Fig. 10, the reactive power output of G7 and G10 at the voltage collapse point increase. For the rest of the generators, the increase of the reactive power output at the voltage collapse point is less than $1 \%$. 
Table 9

The New England 39-bus system generators data.

\begin{tabular}{|c|c|c|c|c|c|c|}
\hline & \multirow[t]{2}{*}{ Bus } & \multirow[t]{2}{*}{$P_{g}^{0}(\mathrm{MW})$} & \multirow[t]{2}{*}{$P_{g}^{\max }(\mathrm{MW})$} & \multicolumn{3}{|c|}{ Capability curve } \\
\hline & & & & $\bar{I}_{f}(\mathrm{pu})$ & $\bar{I}_{a}(\mathrm{pu})$ & $X_{S}(\mathrm{pu})$ \\
\hline Gen 1 & 30 & 250 & 402.5 & 1.3294 & 4.4275 & 0.1000 \\
\hline Gen 2 & 31 & - & 747.5 & 2.9939 & 8.2225 & 0.2950 \\
\hline Gen 3 & 32 & 650 & 920 & 3.1132 & 10.1200 & 0.2495 \\
\hline Gen 4 & 33 & 632 & 862.5 & 3.0358 & 9.4875 & 0.2620 \\
\hline Gen 5 & 34 & 508 & 747.5 & 5.9055 & 8.2225 & 0.6700 \\
\hline Gen 6 & 35 & 650 & 625 & 3.0358 & 9.4875 & 0.2540 \\
\hline Gen 7 & 36 & 560 & 862.5 & 3.4053 & 9.4875 & 0.2950 \\
\hline Gen 8 & 37 & 540 & 805 & 3.2132 & 8.8550 & 0.2900 \\
\hline Gen 9 & 38 & 830 & 1035 & 3.0745 & 11.3850 & 0.2106 \\
\hline Gen 10 & 39 & 1000 & 1380 & 1.1942 & 15.1800 & 0.0200 \\
\hline
\end{tabular}

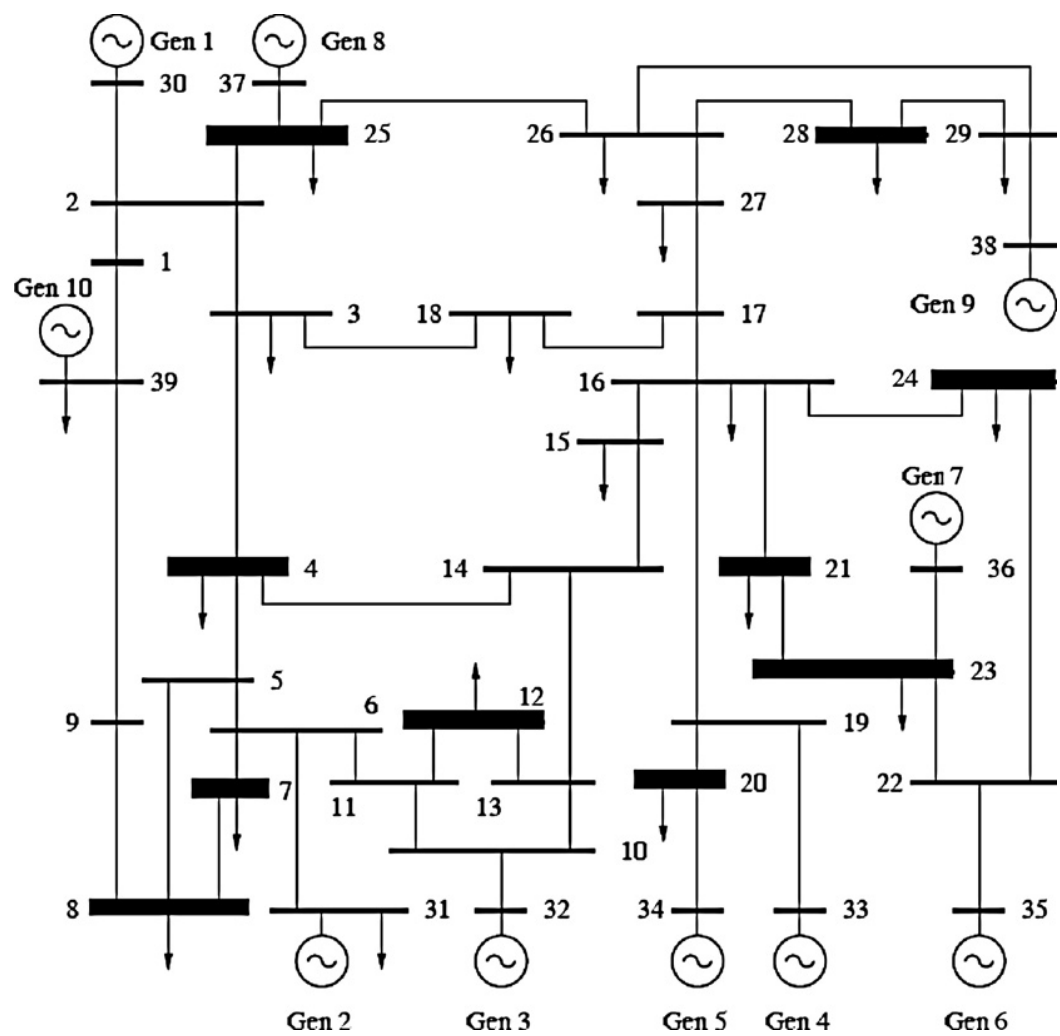

Fig. 7. One line diagram of the New England 39-bus system.

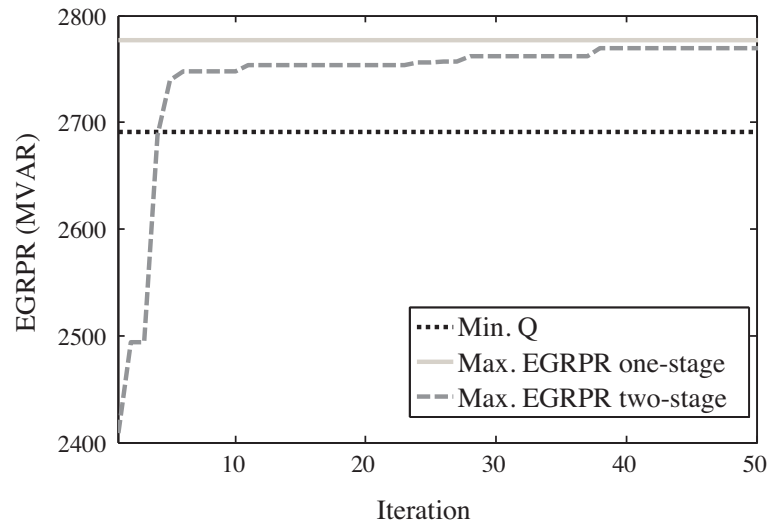

Fig. 8. Sum of all generators' EGRPR for 39-bus system.

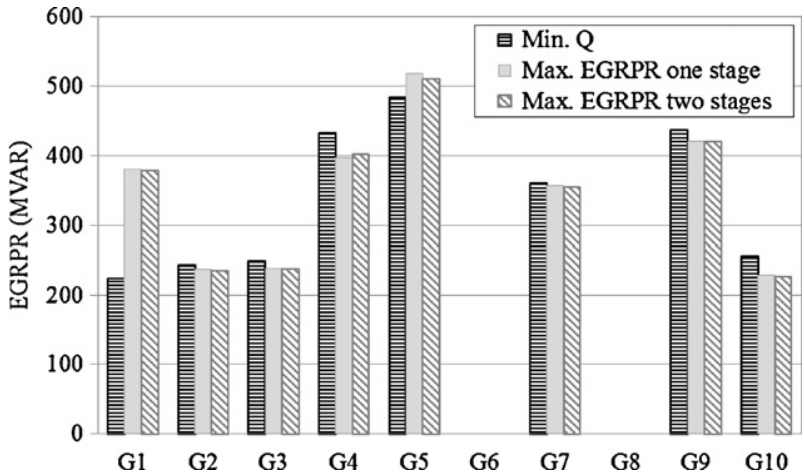

Fig. 9. EGRPR's of each generator for different optimization approaches for New England 39-bus system. 


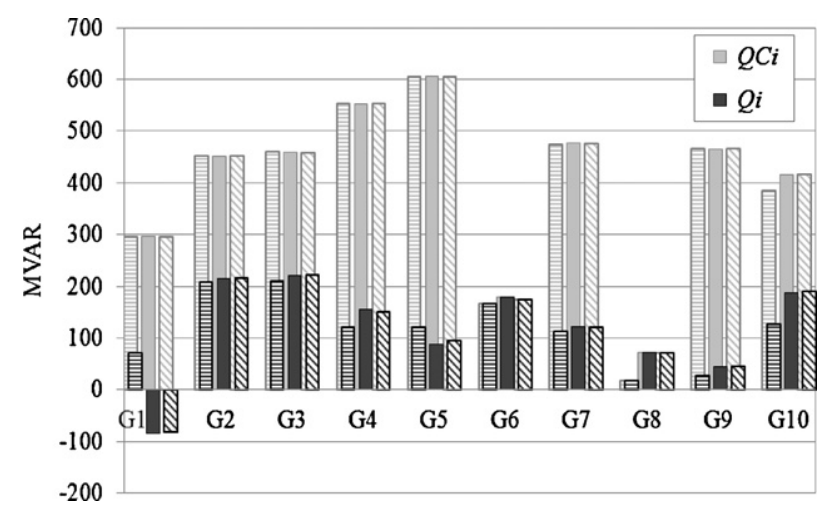

Fig. 10. Generated reactive power of each generator at the current operating point and the voltage collapse point for different optimization approaches for New England 39-bus system.

\subsection{Discussion on the convergence of two-stage method}

The main specification of the two-stage approach in comparison to the one-stage approach is that it deals with an adjustment of the operating point and a smaller optimization problem to derive the collapse points in an iterative manner. The problem solution related to the collapse point is more time consuming since it refers to the complementarity constraints. The simulation results in the 6-bus system demonstrate that the solution of the two-stage approach is faster than one-stage approach, while in the 39-bus system it is the contrary. As a result in this optimization, the number of iterations offsets the advantage of the decomposition, particularly when the power system size increases.

\section{Conclusion}

The voltage and reactive power scheduling along with RPR management are proposed as a convenient preventive countermeasure against voltage instability. The suggested optimization method maximizes EGRPR to improve the VSM at the pilot nodes using onestage or two-stage approaches. For the small systems, two-stage approach is more efficient than one-stage, but it is not effective in larger study cases, since it iteratively solves a large problem at the voltage collapse point. The solutions are compared to the result of the minimization of the generators reactive power output as the reference case.

Owing to the detailed modeling of the generators reactive power limits and the switch mode that allows them to increase their reactive power output at the collapse point, the EGRPR does not increase significantly in the system with PV generators only. But in the system with PQ generators, this optimization effectively increases the EGRPR.

Contingency analysis demonstrates that the necessity of voltage and reactive power rescheduling as corrective actions are reduced. However, some contingencies need the corrective actions in the form of active power scheduling or load shedding because the optimization is not effective in these cases. Therefore, the proposed method ensures the maximum attainable preventive security margin from the available voltage and reactive power control resources without any change in the active power schedule. This can distinguish the effective RPR of generators in the system that can be utilized in the realization of reactive power markets and unbundled reactive power support services. Furthermore, since this optimization method takes into account the role of both PV and PQ generators, it can be extended for the systems with other voltage and reactive power control devices.

\section{Acknowledgements}

The authors thankfully acknowledge Swiss Electric Research as the results reported in this paper have been carried out within the framework of the research project "Security of Multi-Area Power Systems (MARS)".

\section{References}

[1] O. Alizadeh Mousavi, R. Cherkaoui, "Literature survey on fundamental issues of voltage and reactive power control", October 2011 [Online]. Available at: http://mars.ethz.ch/en/researh-and-publications/publications.html

[2] T.V. Cutsem, C. Vournas, Voltage Stability of Electric Power Systems, Kluwer Acad. Publ., Boston/London/Dordrecht, 1998.

[3] H. Song, B. Lee, S.H. Kwon, V. Ajjarapu, Reactive reserve-based contingency constrained optimal power flow (RCCOPF) for enhancement of voltage stability margins, IEEE Transactions on Power Systems 18 (4) (2003) 1538-1546.

[4] B.H. Chowdhury, C.W. Taylor, Voltage stability analysis: $V-Q$ power flow simulation versus dynamic simulation, IEEE Transactions on Power Systems 15 (4) (2000) 1354-1359.

[5] D. Feng, B.H. Chowdhury, M.L. Crow, L. Acar, Improving voltage stability by reactive power reserve management, IEEE Transactions on Power Systems 20 (1) (2005) 338-345.

[6] A. Rabiee, MVAR management using generator participation factors for improving voltage stability margin, Journal of applied sciences 9 (11) (2009) 2123-2129.

[7] T.V. Menezes, L.C.P. da Silva, V.F. da Costa, Dynamic VAR sources scheduling for improving voltage stability margin, IEEE Transactions on Power Systems 18 (2) (2003) 969-971.

[8] T.V. Menezes, L.C.P. da Silva, C.M. Affonso, V.F. da Costa, MVAR management on the pre-dispatch problem for improving voltage stability margin, IEE Proceedings Generation, Transmission \& Distribution 151 (6) (2004) 665-672.

[9] F. Capitanescu, Assessing reactive power reserves with respect to operating constraints and voltage stability, IEEE Transactions on Power Systems 26 (4) (2011) 2224-2234.

[10] B. Lee, Y.H. Moon, H. Song, Reactive optimal power flow incorporating margin enhancement constraints with nonlinear interior point method, IEE Proceedings Generation, Transmission \& Distribution 152 (6) (2005) 961-968.

[11] F. Capitanescu, T. Van Cutsem, Preventive control of voltage security margins: a multi-contingency sensitivity-based approach, IEEE Transactions on Power Systems 17 (2) (2002) 358-364.

[12] R.A. Schlueter, A voltage stability security assessment method, IEEE Transactions on Power Systems 13 (4) (1998) 1423-1438.

[13] M. Zima, D. Ernst, On multi-area control in electric power systems, in: Proc. of PSCC, 2005.

[14] Y. Phulpin, M. Begovic, M. Petit, J.B. Heyberger, D. Ernst, Evaluation of network equivalents for voltage optimization in multi-area power systems, IEEE Transactions on Power Systems 24 (2) (2009) 729-743.

[15] P.A. Ruiz, P.W. Sauer, Reactive power reserve issues, in: Proceeding of North American Power Symposium, Carbondale, 2006, pp. 539-545.

[16] O. Alizadeh Mousavi, M. Bozorg, A. Ahmadi-Khatir, R. Cherkaoui, Reactive power reserve management: preventive countermeasure for improving voltage stability margin, in: IEEE Power Eng. Soc. Gen. Meet., 2012.

[17] R. Ramanathan, C.W. Taylor, BPA reactive power monitoring and control following the August 10, 1996 power failure, in: Proceeding of VI-th SEPOPE Conference, 1998

[18] F. Capitanescu, T. Van Cutsem, Evaluation of reactive power reserves with respect to contingencies, in: Bulk Power Syst. Dyn. and Control V, 2001.

[19] L. Bao, Z. Huang, W. Xu, On-line voltage stability monitoring using var reserves, in: IEEE Power Eng. Soc. Gen. Meet., 2003, p. 1754.

[20] B. Leonardi, V. Ajjarapu, Investigation of various generator reactive power reserve (GRPR) definitions for online voltage stability/security assessment, in: Power and Energy Soc. Gen. Meet. - Convers. and Deliv. of Electr. Energy in the 21st Century, 2008, pp. 1-7.

[21] A.E. Efthymiadis, Y.I. Guo, Generator reactive power limits and voltage stability, Power System Control, Operation and Management. Fourth International Conference on Power System Control and Management (1996) 196-199.

[22] W. Rosehart, C. Roman, A. Schellenberg, Optimal power flow with complementarity constraints, IEEE Transactions on Power Systems 20 (2) (2005) 822-843.

[23] S. Boyd, A. Mutapcic, Subgradient Methods Notes for EE364b, Stanford University, 2008, April.

[24] A.J. Conejo, E. Castillo, R. Minquez, Decomposition techniques in mathematical programming, Engineering and Science Applications (2006).

[25] T. Amraee, A. Soroudi, A.M. Ranjbar, Probabilistic determination of pilot points for zonal voltage control, IET Generation, Transmission \& Distribution 6 (1) (2012) 1-10.

[26] T. Amraee, A.M. Ranjbar, R. Feuillet, Immune-based selection of pilot nodes for secondary voltage control, European Transactions on Electrical Power 20 (7) (2010) 938-951.

[27] S. Mei, X. Zhang, M. Cao, Power Grid Complexity, Springer, Beijing, 2011.

[28] G.L. Torres, V.H. Quintana, An interior point method for nonlinear optimal power flow using voltage rectangular coordinates, IEEE Transactions on Power Systems 13/4 (1998) 1211-1218. 